\title{
Proton-implanted optical waveguide detectors in GaAs
}

\author{
H. Stoll and A. Yariv \\ California Institute of Technology, Pasadena, California 91109
}

\section{R. G. Hunsperger and G. L. Tangonan}

Hughes Research Laboratories, Malibu, California 90265

(Received 17 August 1973)

\begin{abstract}
Defect levels introduced by implanting GaAs with high-energy protons give rise to optical absorption at wavelengths greater than that of the normal absorption edge at $0.9 \mu$. Optical waveguide detectors may be fabricated by taking advantage of this absorption mechanism in the presence of a Schottky barrier depletion layer. Detector response times less than $200 \mathrm{~ns}$ and external quantum efficiencies of $16 \%$ have been observed.
\end{abstract}

In a continuing program to build circuit components for integrated optics we have investigated the possibility of fabricating optical detectors by proton implantation of GaAs dielectric waveguides. The basic principle is as follows: A small volume of an epitaxial GaAs waveguide, such as the one shown in Fig. 1, is implanted with protons. Structural disorder created by the implantation process causes the previously low-loss waveguide to become highly lossy for radiation around $1 \mu \mathrm{m}$. One of the mechanisms responsible for this absorption is the liberation of free carriers which had become trapped at defect centers. A photodetector results when these carriers are swept through the depletion layer generated by a reverse-biased Schottky barrier which has been deposited over the implanted region as shown in Fig. 1. We report below the fabrication of integrated optical detectors based on this principle. The detectors have been found to be sensitive to light of wavelengths $>0.9$ $\mu \mathrm{m}$, a wavelength which can be transmitted nearly unattenuated by the GaAs guides used. The optical waveguide structure consisted of a $3.5-\mu \mathrm{m}$-thick $n$-type (S-doped, $n \simeq 10^{16} / \mathrm{cm}^{3}$ ) epitaxial film grown on a degenerate $n$-type substrate $\left(n \simeq 1.25 \times 10^{18} / \mathrm{cm}^{3}\right)$. The guidesubstrate refractive index discontinuity generated by the plasma depression effect allowed good optical confinement for the particular guide thickness used ${ }^{1}$; prior to proton implantation optical attenuation at $1.15 \mu \mathrm{m}$ was measured to be $1.3 \mathrm{~cm}^{-1}$ and could be accounted for by consideration of free carrier substrate penetration losses.

As shown in Fig. 1, a small volume of the waveguide

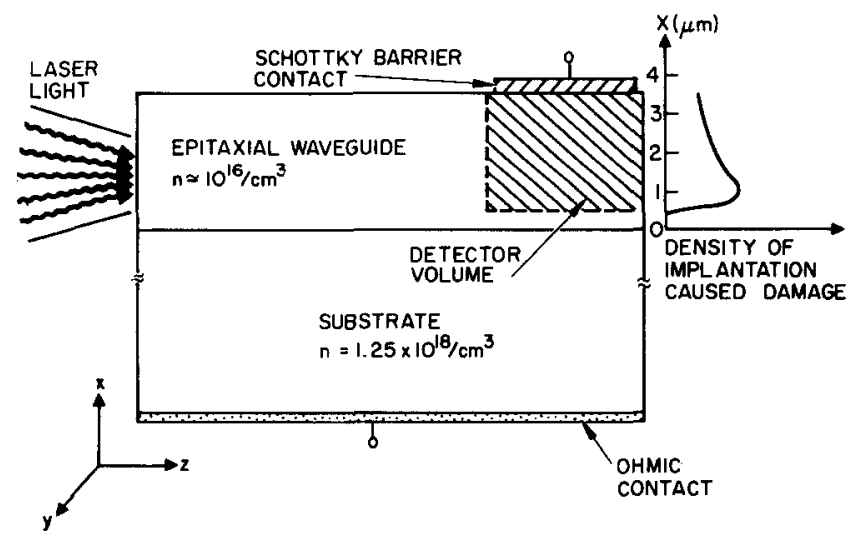

FIG. 1. Diagram of proton-implanted optical waveguide detector. structure was proton bombarded to form the waveguide detector; $300-\mathrm{keV}$ protons were used, the total integrated flux of which was $2 \times 10^{15} / \mathrm{cm}^{2}$. The damage layer which resulted was approximately $3 \mu \mathrm{m}$ thick with a damage peak occurring about $2.5 \mu \mathrm{m}$ below the surface.

The implanted waveguide was then annealed at $500^{\circ} \mathrm{C}$ for $30 \mathrm{~min}$ in order to allow some optical transmission through the damaged region; residual losses in this region were measured to be $\approx 15 \mathrm{~cm}^{-1}$ based on a comparison of the optical attenuation before and after implantation and annealing. Finally, 11-mil-square $\mathrm{Al}$ Schottky barriers were evaporated in a waffle pattern over the implanted area.

The principle of the operation of this device is similar to that of conventional $p-n$ or $p-i-n$ junction photodetectors.

Upon the application of a reverse bias to the Schottky barrier, a depletion layer is produced which, given sufficient reverse bias, extends across the high-resistivity waveguiding layer to the lower-resistivity substrate. Any dipole transitions made possible by radia-

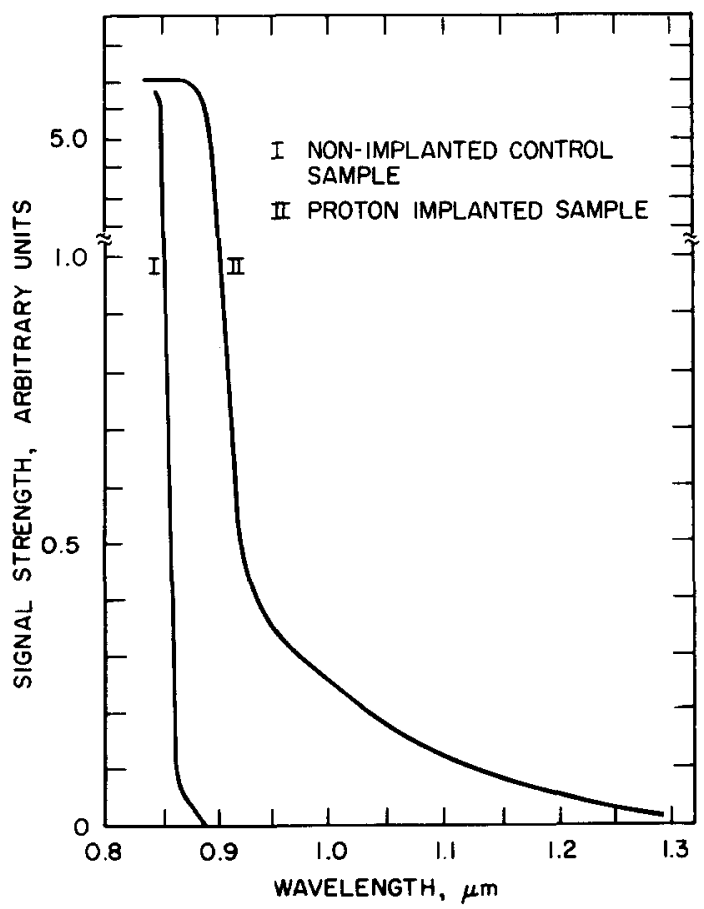

FIG. 2. Photoresponse of optical waveguide detector as a function of wavelength. 


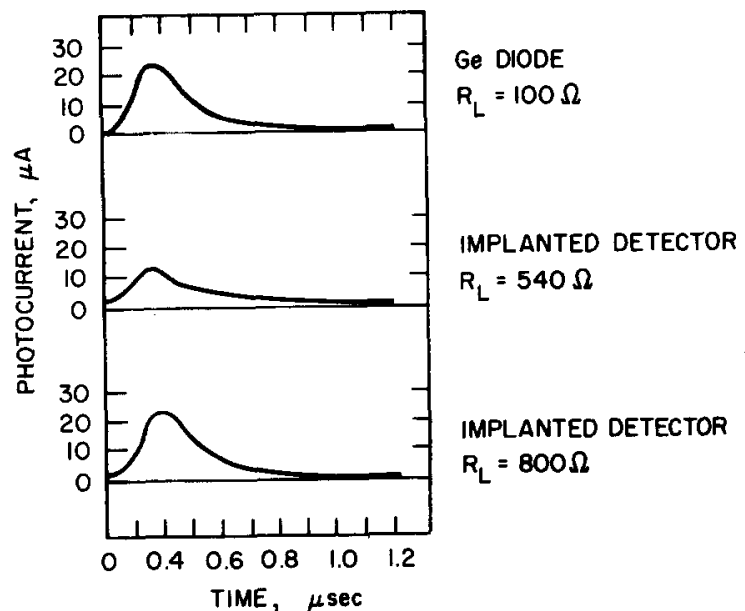

FIG. 3. Response of waveguide detector to pulsed optical signal.

tion-produced defect levels generate free carriers which are swept out of the depletion layer, thereby causing current to flow through an external circuit. By ensuring that the radiation-induced damage extends over most of the waveguiding layer and choosing epitaxial material of high purity, maximum efficiency can be obtained over a given interaction length.

Measurements of the photosensitivity of both unimplanted and implanted annealed samples are shown in Fig. 2. Transparent Au barriers, sputter deposited on the surface, were used to measure sensitivity as a function of wavelength. A monochromator-filtered incandescent light source was focused through the trans parent $\mathrm{Au}$ layer into the active volume of the detectors. The curve for the irradiated sample reveals a defectassociated energy level distribution within the band gap, as well as a shift of the effective absorption edge to lower energies. Calorimetric measurements ${ }^{2}$ made on proton-irradiated GaAs at $1.06 \mu \mathrm{m}$ indicate that substantially all of the implantation-induced optical attenuation in the $1-\mu \mathrm{m}$-wavelength region can be attributed to absorption as opposed to diffuse scattering. The origin of this absorption is thought to be disorder-induced band tailing. The possibility that it is due, at least in part, to the excitation of electrons from energy levels associated with As (or $\mathrm{Ga}$ ) vacancy complexes is strong, particularly in view of the fact that free carrier compensation by proton implantation appears to be Fermilevel dependent. ${ }^{3,4}$

An attempt was made to measure detector rise and fall times at $1.06 \mu \mathrm{m}$ using an acoustically $Q$-switched Nd: YAG laser focused into a cleaved face of the waveguide structure of Fig. 1. The pulse response of the implanted detector is compared to that of a commercial Ge (Philco L4521) detector in Fig. 3. In both cases the

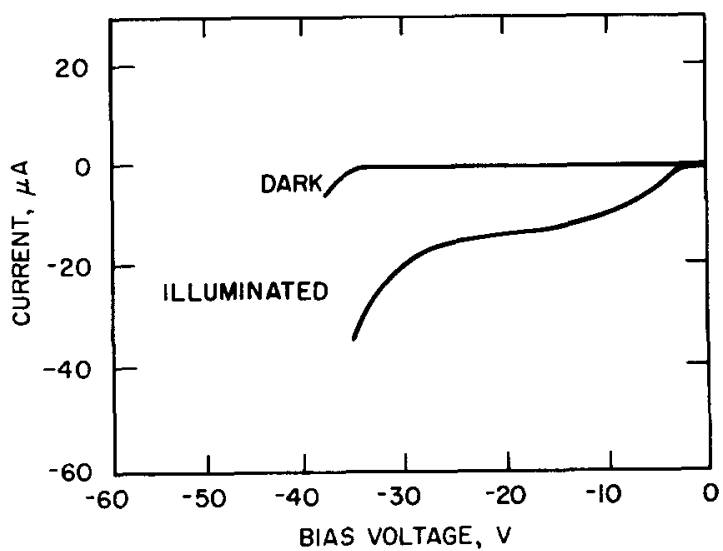

FIG. 4. Reverse bias current-voltage characteristics of waveguide detector.

rise and fall times appeared to be $\sim 200$ and $\sim 400 \mathrm{~ns}$, respectively. Reducing the size of the load resistors used merely decreased the signal strength; the pulse shapes remained the same. Since the Ge detector response is in the $\mathrm{GHz}$ range, it was concluded that the rise and fall times measured were a function of the $Q$ switch ed laser and did not represent limits of the implanted detector.

Figure 4 shows $I-V$ curves for a reverse-biased implanted detector under darik and $1.15-\mu \mathrm{m}$ illumination conditions. The measured external quantum efficiency at $1.15 \mu \mathrm{m}, 294^{\circ} \mathrm{K}$, and a reverse bias of $20 \mathrm{~V}$ was about $16 \%$. This number should increase as optimal proton damage profile and heat treatment conditions are approached for a given waveguiding configuration.

In conclusion we have demonstrated a simple technique for fabricating integrated optical detectors sensitive at wavelengths $>0.9 \mu \mathrm{m}$ using proton implantation. Device performance is promising and should improve as implantation and heat treatment procedures are refined.

The authors would like to thank Dr. W.C. Holton of Texas Instruments for providing the epitaxial GaAs material.

${ }^{1}$ D. Hall, A. Yariv, and E. Garmire, Appl. Phys. Lett. 17, 127 (1970).

${ }^{2} \mathrm{H}$.J. Stein, Proceedings of the International Conference on Ion Implantion in Semiconductors and Other Materials, Yorktown Heights, 1972 (Plenum, New York, 1973).

${ }^{3}$ H. Stoll, E. Garmire, A. Yariv, and R.G. Hunsperger (unpublished).

${ }^{4} \mathrm{~K}$. Wohlenben and W. Beck, Z. Naturforsch. 219, 1057 (1966). 